\title{
RAMAN SPECTRA OF TWO SAMPLES OF RUBRENE LAYERS
}

\author{
Magdaléna Kadlečíková * — Juraj Breza * - Jozef Liday * \\ Helmut Sitter $^{* *}$ — Shaima Abd Al-Baqi ${ }^{* *}$
}

\begin{abstract}
This experimental work deals with measuring Raman spectra of rubrene. The objective is to optimize the measurement procedure of rubrene layers on a substrate. The main outcome of the work is identification of rubrene and of the singlecrystalline nature of the measured spots of the rubrene layer.

K e y w or d s: rubrene, Raman spectroscopy
\end{abstract}

\section{INTRODUCTION}

Raman spectroscopy is a very powerful tool to characterize the optical, chemical and structural properties of a layer of small organic molecules in a non-destructive way. The detected vibration modes of the molecules act as fingerprints for the observed material, which allows detecting the different constituents of thin organic films. Assuming a pure material, the Raman spectra will show the very distinguished breathing and vibration modes of the molecule. Since these modes are very sensitive to any change in the structure of the molecule, they can be used to study chemical reactions or polymerization processes caused by the fabrication of the layers or by the handling processes employed to fabricate devices out of them. In addition, strain and stress states in thin films can be detected from the line shifts in the Raman spectra, which is very important for the performance and stability of electronic devices made of such layers.

Rubrene is one of the most promising organic materials with respect to its high carrier mobility. However, these spectacular results were so far only found in devices fabricated from single crystals of rubrene. All devices made from thin layers of rubrene have exhibited carrier mobility values lower by orders of magnitude. Nevertheless, it is a big challenge to grow rubrene thin films with approximately the same carrier mobilities as those of single crystals.

The method of choice for such growth experiments is the so-called hot wall epitaxy [1] because it works close to thermodynamic equilibrium and therefore should provide highly ordered organic layers which could lead to high carrier mobility as the number of scattering centres is clearly reduced in such layers.

\section{EXPERIMENTAL}

The rubrene layers were grown at the Johannes Kepler University in Linz and Raman investigations were carried out at the Slovak University of Technology in Bratislava. Micro-Raman measurements of the deposited materials (layers) were performed using an ISA Dilor-Jobin YvonSpex Labram confocal system with $632.8 \mathrm{~nm}$ radiation from a He-Ne laser using a back-scattering geometry. Microscope objectives $50 \times$ and $100 \times$ were used to focus the laser beam onto a spot of approximately $10 \mu \mathrm{m}$ in diameter (as seen in Fig. 3b), and to collect the scattered light which then passed through the spectrometer onto a CCD detector. Furthermore, a confocal hole with a diameter of $800 \mu \mathrm{m}$, a spectrograph entrance slit of 200 $\mu \mathrm{m}$, and 1800 grooves $/ \mathrm{mm}$ diffraction grating were employed. All measurements were performed at room temperature. Calibration measurements performed on singlecrystalline $\mathrm{Si}(100)$ proved a measurement uncertainty of $\pm 2 \mathrm{~cm}^{-1}$ in comparison with the catalogue value for silicon $520.7 \mathrm{~cm}^{-1}$.

We have carried out test measurements of Raman spectra of samples S27 and S28 at places that are seen in the middle of the fields shown in photographs Fig. 1 to Fig. 3.

Altogether, 12 spots have been measured on every sample in the spectral range from 200 to $3000 \mathrm{~cm}^{-1}$. Selected Raman spectra are shown in Figs. 4 to 7 . No other types of spectra have been observed. After optimizing the measuring conditions the measurement window has been chosen from 1000 to $1700 \mathrm{~cm}^{-1}$. So as not to mask experimental details, the measured Raman spectra have not been processed mathematically or smoothed. Similarly, no background subtraction has been performed or fitting by Gaussian or Lorentzian curves. Each of the Raman spectrum lies on a high photoluminescence spectrum.

The Raman bands which have been measured in the Raman spectra are summarized in Table 1.

Department of Microelectronics, Faculty of Electrical Engineering and Information Technology, Slovak University of Technology, Ilkovičova 3, 81219 Bratislava, Slovakia, juraj.breza@stuba.sk; ${ }^{* *}$ Institute of Semiconductor and Solid State Physics, Johannes Kepler University of Linz, A-4040 Linz, Austria 


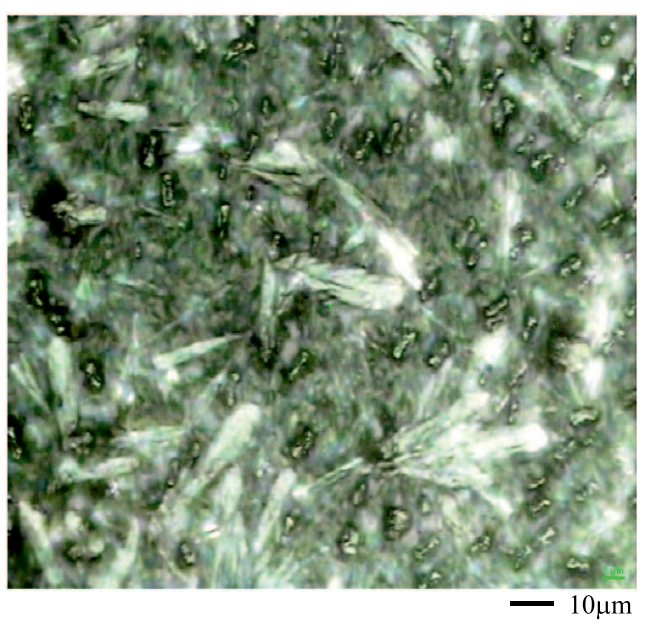

Fig. 1. Sample S27, spot of collecting the Raman spectrum

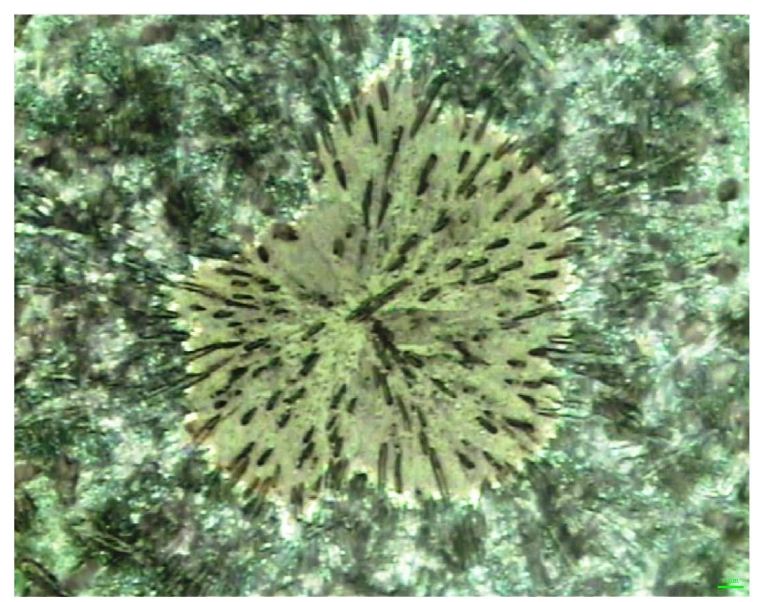

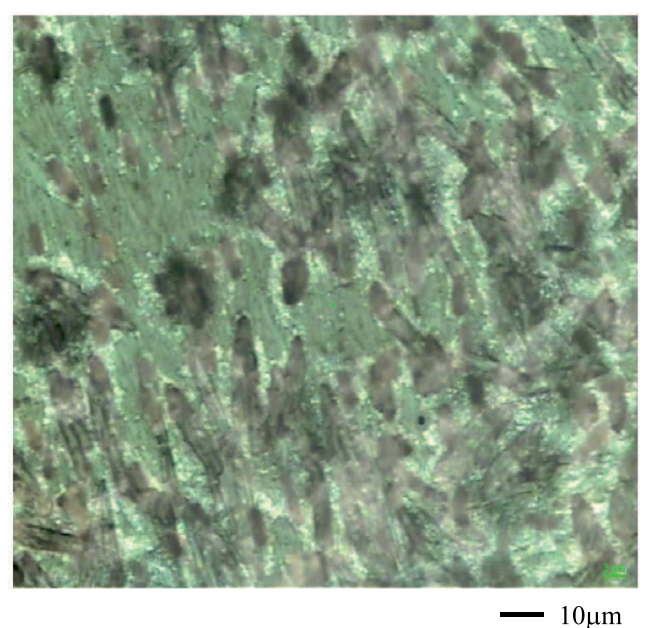

Fig. 2. Sample S28 1, spot of collecting the Raman spectrum

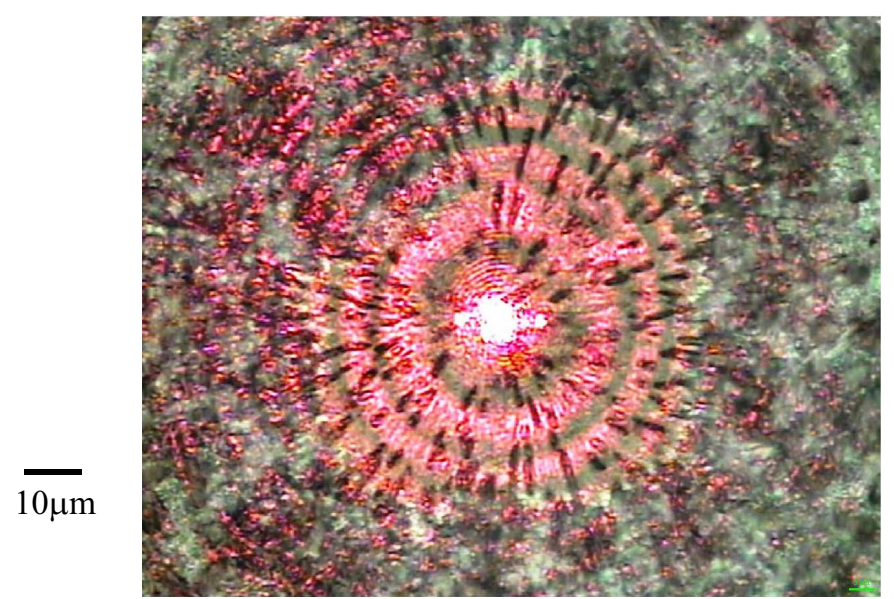

Fig. 3. (a) - Sample S28 2, spot of collecting the Raman spectrum focused on needle-structured crystalline formations on (or inside) the film, (b) - Sample S28 2, the spot excited by the He-Ne laser. The spot of the beam that is seen in the middle of the picture has a diameter of 8 to 10 micrometers

Table 1. Positions of the bands in Raman spectra

\begin{tabular}{cccc}
\hline $\begin{array}{c}\mathrm{S} 27 \\
\left( \pm 2 \mathrm{~cm}^{-1}\right)\end{array}$ & $\begin{array}{c}\mathrm{S} 28 \mathrm{1} \\
\left( \pm 2 \mathrm{~cm}^{-1}\right)\end{array}$ & $\begin{array}{c}\mathrm{S} 282 \\
\left( \pm 2 \mathrm{~cm}^{-1}\right)\end{array}$ & $\begin{array}{c}\text { Peak positions * } \\
\left(\mathrm{cm}^{-1}\right)\end{array}$ \\
\hline 1003 & 1003 & 1004 & 1003.9 \\
& & & 1046.0 \\
1167 & & 1168 & 1163.0 \\
1216 & & & \\
& & 1302 & 1268.2 \\
1302 & & 1311 & 1399.9 \\
1311 & & & 1315.9 \\
& & $\mathbf{1 4 3 6}$ & $\mathbf{1 4 3 2 . 3}$ \\
$\mathbf{1 4 3 4}$ & & $\mathbf{1 5 2 1}$ & $\mathbf{1 5 1 9 . 9}$ \\
$\mathbf{1 5 2 1}$ & & $\mathbf{1 5 4 0}$ & $\mathbf{1 5 3 9 . 9}$ \\
$\mathbf{1 5 4 1}$ & & & \\
& 1605 & $\mathbf{1 6 1 6}$ & $\mathbf{1 6 1 8 . 8}$ \\
$\mathbf{1 6 1 6}$ & &
\end{tabular}

* in Raman spectrum of rubrene [2]

\section{CONCLUSION}

The analysis of the Raman spectra is based on knowing the chemical structure of rubrene. Rubrene crystals are molecular crystals in which the molecules are loosely bound to each other via van der Waals forces and may create different crystalline structures. Polymorphism is a phenomenon common to many organic crystals.

The measured Raman spectra of samples S27 and S28 2 are in good agreement with experimental results and calculations given, eg in [2] and point at the singlecrystalline nature of the measured spots of the rubrene layer.

The Raman spectrum S 281 does not confirm the single-crystalline nature of the examined spot of the sample. In the Raman spectrum of sample S28 1 only one band is identified, its middle being located at $\approx 1003$ $\mathrm{cm}^{-1}$, and a weak band at $\approx 1605 \mathrm{~cm}^{-1}$. The band centred at $\approx 1605^{-1}$ has been observed only in the Raman spectrum of sample S28 1. 


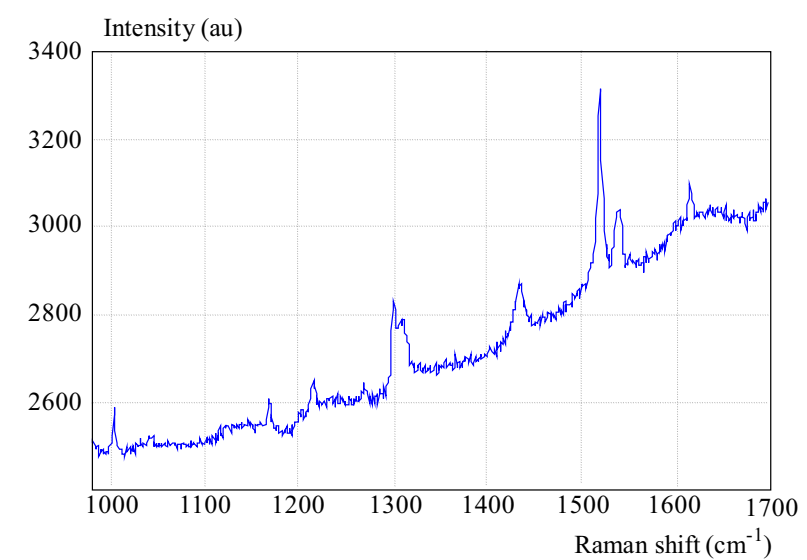

Fig. 4. Raman spectrum of sample $\mathrm{S} 27$ at spot 1 in the spectral window 1000 to $1700 \mathrm{~cm}^{-1}$

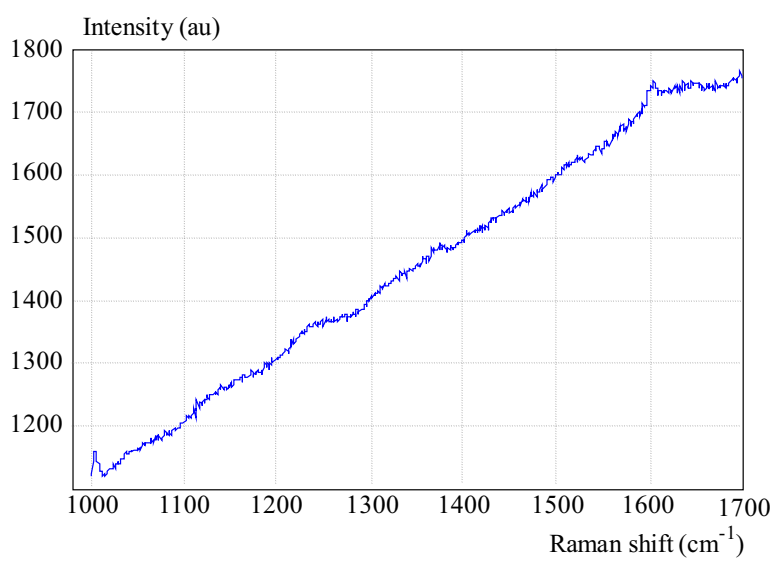

Fig. 6. Raman spectrum of sample S28 at place 1 in the spectral range from 1000 to $1700 \mathrm{~cm}^{-1}$.

The band centred at $\approx 1003 \mathrm{~cm}^{-1}$ is typical for phenyl groups [3] present in all measured Raman spectra.

The bands centred at $\approx 1434 \mathrm{~cm}^{-1}, \approx 1521 \mathrm{~cm}^{-1}$, $\approx 1541 \mathrm{~cm}^{-1}$ and $\approx 1616 \mathrm{~cm}^{-1}$ (shown bold in Table 1.) in the Raman spectra of samples $S 27$ and $S 282$ are related to the tetracene nuclei of the molecules [5].

The Raman spectra in Figs. 4 and 7 unambiguously identify rubrene. The Raman bands at $\approx 1302 \mathrm{~cm}^{-1}$ and $\approx 1311 \mathrm{~cm}^{-1}$ (shown in italic in Tab. 1), after comparing them with the Raman spectra of phenyl and tetracene [5], are decisive for the crystalline form of rubrene (the type of structure to be determined by another method). This result is the most important outcome of our part of the experiment.

From the microscopic point of view, the tested samples have a relief nature. Therefore it is not possible to record Raman maps of the samples. In single points, however, one can identify rubrene by Raman spectroscopy. Evaluation of the Raman spectra requires a deeper knowledge of the process of preparation technology, including the precursors, and comparisons with other methods.

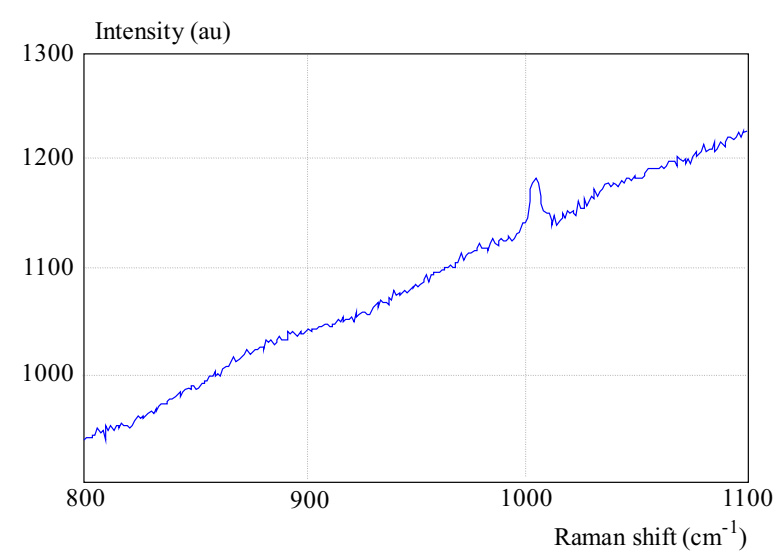

Fig. 5. Band approx $1003 \mathrm{~cm}^{-1}$ in the Raman spectrum of sample S28, measured at point 1

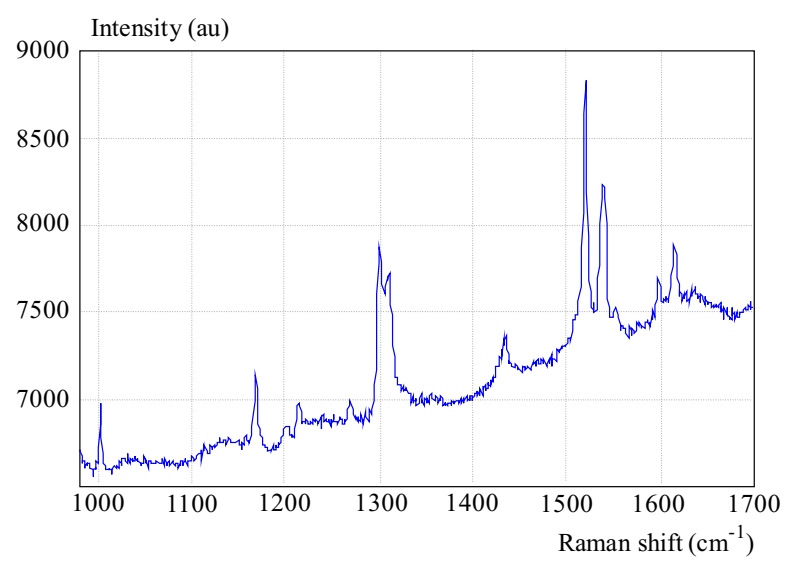

Fig. 7. Raman spectrum of sample S28 at place 2

\section{Acknowledgement}

This work has been supported by grants Action Austria-Slovakia No. 63s7 and VEGA 1/0746/09 of the Ministry of Education of the Slovak Republic.

\section{REFERENCES}

[1] SITTER, H.: Organic Nanostructures for Next Generation Devices, Hot-Wall Epitaxial Growth of Films of Conjugated Molecules, Chapter 5, Edited by K. Al-Shamery, H.-G. Rubahn and H. Sitter. Springer-Verlag Berlin Heidelberg 2008.

[2] WEINBERG-WOLF, J. R.-McNEIL, L. E.-LIU, S.-KLOC, CH. : Evidence of Low Intermolecular Coupling in Rubrene Single Crystals by Raman Scattering, J. Phys.: Condens. Matter 19 (2007), 276204 (15pp), doi: 10.1088/0953-8984/19/27/276204.

[3] DAVIS, C. A.-RUPENG, Z.-CROSSLEY, J. A. A.-GRAVES, P. R.-MYHRA, S. : Identification by Raman Microprobe (LRM) of Phenyl Groups in Thin Surface Coatings, Applied Surface Science 65/66 (1993), 362-365.

[4] http://pubchem.ncbi.nlm.nih.gov/summary/summary.cgi?cid $=68203$.

[5] http://www.sigmaaldrich.com/sigma-aldrich/home.html.

Received 6 March 2010 